\title{
Post-Cesarean Section Hemorrage Treated by a Collagen Patch Coated with the Human Coagulation Factors
}

\section{Andrea Tinelli*}

Division of Experimental Endoscopic Surgery, Imaging, Minimally Invasive Therapy \& Technology, Department of Obstetrics and Gynaecology, Vito Fazzi Hospital, Piazza Muratore, 73100 Lecce, Italy

\begin{abstract}
Uterine hemorrhage is a common problem at the time and after caesarean section especially in a repeat caesarean section, where the surgical techniques used widely differ. An inadequate hemostasis of the uterine incision and the vesical-uterine vessels during CS, in closed and open visceral peritoneum, could lead to early hemorrhagic compliances, to control by further surgery.

We present the case of a 31 year old woman undergoing a scheduled repeat emergency caesarean section. She had progressive bleeding from the incision site during the first post-operative days, since in third day she developed a hemorrhagic shock for a hemoperitoneum. Patient was re-operated and bleeding at uterine site incision was not controlled by traditional management including intravenous oxytocin and ergometrine, intramyometrial ergometrin and local hemostatic sutures, but was successfully controlled by equine collagen patch coated with human coagulation factors. Her post operative period was uneventful and she and her baby were discharged in good condition on day 5 . Both of them were doing well on a follow up visit after 1 month.
\end{abstract}

Keywords: Caesarean section; Hemorrhage; Uterine incision; Human coagulation factors; TachoSil; Post-cesarean section hemorrhage; Hemoperitoneum; Drainage; Complications; Hysterectomy; Oxytocins; Prostaglandin

\section{Introduction}

Today one in four or $25 \%$ of women have a cesarean section even if the World Health Organization (WHO) considers the percentage of births by Cesarean Section (CS), a health care quality indicator, providing to protect health of mother and newborn. In 1985 the WHO affirmed that "There is no justification for any region to have CS rates higher than $10-15 \%$ [1].

This percentage will be reconsidered even if no evidence supports the idea that cesareans are as safe as vaginal birth for mother or baby: the increase in cesarean births risks the health and well being of childbearing women and their babies [1].

For elective repeat cesarean, the consensus of dozens of studies totaling tens of thousands of women is that elective repeat cesarean section is riskier for the mother and not any safer for the baby [2].

The surgical techniques used in repeated cesarean section differ from: different types of uterine incision; methods of performing the uterine incision; suture materials and technique of uterine closure (including single versus double layer closure of the uterine incision) on maternal health, infant health, and health care resource use. Moreover there is an additive surgical problem, the risk of re-laparotomy for late post-cesarean section hemorrhage, due to a dehiscence of a lower uterine segment incision [3]. In this case we report a secondary postpartum hemorrhage that occurred 3 days after repeated cesarean section.

\section{Case Report}

A patient of 31 year old underwent a scheduled repeat caesarean section in a Obstetric and Gynecology Department of a University affiliated Hospital in 2011: she had 3 pregnancies in her anamnesis, one recent previous cesarean sections in 2010, a Body Max Index of 28, a previous laparotomy for $12 \mathrm{~cm}$ left ovarian cyst and a laparoscopy for a single myomectomy. Obstetrics agreed with woman to delivery by a scheduled repeated cesarean section, who was at 38 weeks by Stark method [4], by a Joel-Cohen laparotomy, performed by a superficial transverse cut in the cutis, about 3 centimetres below an imaginary line drawing between the iliac anterior-superior spines, cutting only through cutis. A small transverse opening was made in the fascia with the tip of a scalpel, and then, the fascia was opened transversely underneath the fat tissue and blood vessels by pushing the slightly open tip of a pair of straight scissor, first in one direction, and then in the other. The fascia was then stretched caudally and cranially using the index fingers to make room for the next step. The surgeon and his assistant each inserted their index and third fingers under the muscles, and stretched the muscles, blood vessels, and the fat tissue by manual bilateral traction. The peritoneum was then opened transversely using only both index fingers, in order to avoid the bladder that could be shifted upon its anatomical site. Then we cut, by a Munro-Kerr transversal incision, first, the uterine visceral peritoneum and consequently the low uterine segment (LUS). After the fetal extraction, a male baby weighing $3.8 \mathrm{~kg}$ with an APGAR of 9 at $1 \mathrm{~min}$ and 10 at $5 \mathrm{~min}$, and placental removal, a standard Oxytocin drip was started and the injection ergometrine was given intravenously. During the CS we reported a bladder strictly tight to anterior uterine wall; the surgical detachment by Mayo scissors of the bladder from uterus was difficult for the haemostasis of the bladder flap. The LUS was sutured by a continuous single layer polyglactin (vicryl) suture, without the suture of the visceral peritoneum and

*Corresponding author: : Dr. Andrea Tinelli, Division of Experimental Endoscopic Surgery, Imaging, Minimally Invasive Therapy \& Technology, Department of Obstetrics and Gynaecology, Vito Fazzi Hospital, Piazza Muratore, 73100 Lecce, Italy, Tel: +39/339/2074078; Fax: +39/0832/661511; E-mail: andreatinelli@gmail.com

Received December 04, 2011; Accepted December 06, 2011; Published December 15, 2011

Citation: Tinelli A (2011) Post-Cesarean Section Hemorrage Treated by a Collagen Patch Coated with the Human Coagulation Factors. J Clinic Case Reports 1:e103. doi:10.4172/2165-7920.1000e103

Copyright: () 2011 Tinelli A. This is an open-access article distributed under the terms of the Creative Commons Attribution License, which permits unrestricted use, distribution, and reproduction in any medium, provided the original author and source are credited. 
by suturing the parietal peritoneum. The abdominal wall was closed becoming by fascia, without abdominal muscles suturing. After that, patient was sent in obstetric section of ObGyn Department for postpartum normal post-operative hospital stay. In the first two days, the hemoglobin check reported a decrease of more than 1 point for day, from 11.4 to $8.7 \mathrm{mg} / \mathrm{Dl}$, with general normal post-operative condition; then in the morning of third post-operative day, nurses have found a sudden hemorrhagic shock. Clinicians immediately have revived woman with restoration of circulating volume by placement of two large bore IVs, rapid infusion of a balanced crystalloid solution and adequate oxygenation. An immediate adbomino-pelvic ultrasonography (US) in different sections, revealed pathological findings of hemoperitoneum: an $11 \mathrm{~cm}$ free fluid collection in abdominal cavity that reached diaphragm. Hence obstetrics transferred patient in surgical room to perform an urgent re-laparotomy who, despite good approximation, it revealed a hemoperitoneum and a constant oozing of blood from the uterine incision site (Figure 1). The hemoperitoneum was drained and few bladder vessels previously not well hemostatized were detected and sutured. Extra hemostatic sutures were taken at incision site to control local ooze, but bleeding continued (Figure 2). The uterus was moderately contracted after an intravenously oxytocin drip plus injection ergometrine, and uterine massage. An additive ergometrine drip was injected intramyometrially. However, the oozing from the incision site continued. A drug made by an equine collagen patch

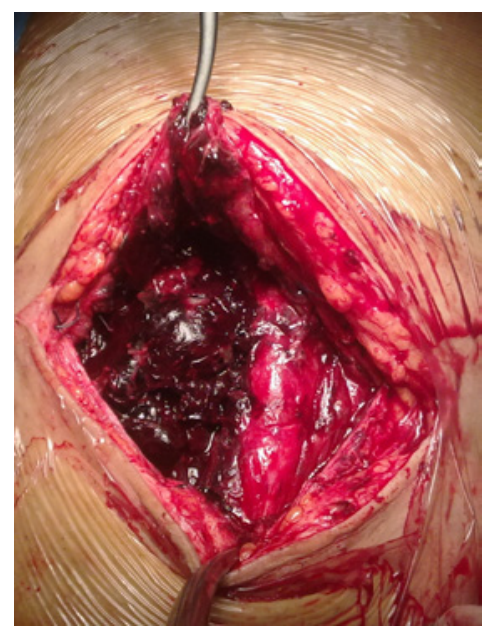

Picture 1: Surgeons performed an urgent re-laparotomy in third-post-operative day, who, despite good approximation, it revealed an haemoperitoneum.

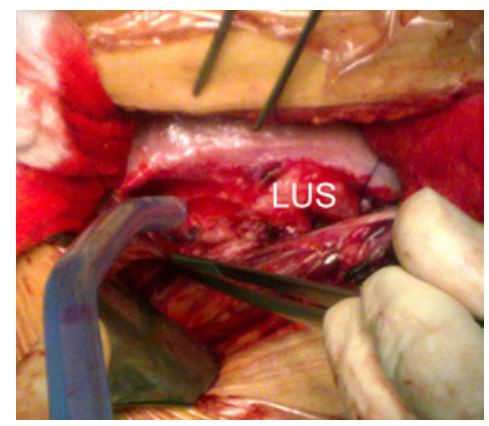

Picture 2: The haemoperitoneum was drained and few bladder vessels previously not well haemostatized were detected and sutured, with extra hemostatic sutures on lower uterine segment (LUS) to control local ooze, but bleeding continued.

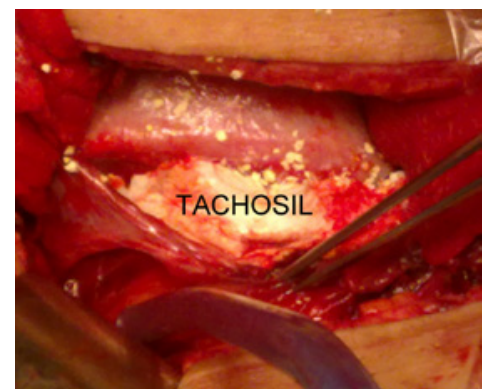

Picture 3: Surgeons placed on the incision site thoroughly wrapping the bleeding site a drug made by an equine collagen patch coated with fibrin glue components, human fibrinogen and human thrombin (TachoSil, Nycomed International Management $\mathrm{GmbH}$, Zurich, Switzerland)

coated with fibrin glue components, human fibrinogen and human thrombin (TachoSil, Nycomed International Management GmbH, Zurich, Switzerland) was placed on the incision site thoroughly wrapping the bleeding site (Figure 3). Bleeding stopped and the site was observed for $10 \mathrm{~min}$ for hemostasis. Finally, at the end of the relaparotomy, a catheter inside the pelvis was placed for drainage. The patient was given intravenous antibiotics and fluids as per the hospital protocol. The clinical conditions, the hematochemical samples, the transabdominal and transvaginal US examinations didn't showed any postoperative complications after the fourth postoperative day, since the pelvic drainage was removed; the patient was discharged in fifth postoperative day in wellbeing, without US signs of intraperitoneal free fluid collections, with normal puerperal uterus size. Patient and her baby were doing well on a follow up visit after 1 month.

\section{Discussion}

Cesarean delivery is now the most common obstetric operation in the world [1], so as in United States, and it has increased dramatically from $5.8 \%$ in 1970 to $32.3 \%$ in 2008 [5]. This rise has not resulted in significant improvement in neonatal morbidity or maternal health. Maternal mortality in the United States has increased from 1998 to 2004 for the increasing incidence of placenta accreta associated with multiple uterine scars requiring the need for emergency cesarean hysterectomy, blood transfusion, and maternal mortality due to obstetric hemorrhage [5]. In a scientific literature review on PubMed there are many articles about the non-closure of the visceral and parietal peritoneum in CS; visceral and parietal peritoneum are still two different anatomical entities and they provoke different early and late complications, including post-partum hemorrhage [6,7].

Complications during and after the surgery include surgical injury to the bladder, uterus and blood vessels (2 per 100), hemorrhage (1 to 6 women per 100 require a blood transfusion), anesthesia accidents, blood clots in the legs ( 6 to 20 per 1000), pulmonary embolism ( 1 to 2 per 1000), paralyzed bowel (10 to 20 per 100 mild cases, 1 in 100 severe), and infection (up to 50 times more common) $[8,9]$.

A retrospective observational study set in a teaching institution in Kolkata, India, showed 66 cases requiring re-laparotomy following 12967 CSs: post-partum hemorrhage in 28 cases (42.4\%) and rectus sheath hematoma in 18 cases $(27.3 \%)$ were the leading indications for re-laparotomy. Of these 66 cases, 63 (95.5\%) had intrapartum cesarean delivery, while three $(4.5 \%)$ had an elective operation. Procedures undertaken at relaparotomy were resuturing of uterine incision in 22 cases $(33.3 \%)$, uterine artery ligation in 19 cases $(28.8 \%)$, and 
drainage of hematoma in 18 cases $(27.3 \%)$. A third laparotomy was needed in 13 cases $(19.6 \%)$, of which 11 were due to secondary postpartum hemorrhage. There were eight maternal deaths following relaparotomy [10].

Another retrospective descriptive survey on 6120 CSs (17\%) out of a total of 36,010 deliveries revealed a re-laparotomy in 44 patients $(0.7 \%)$ of the CSs. The indications were: hemorrhage from uterine atony, hemorrhage from placental bed after operation for placenta previa, uterine sepsis with hemorrhage, hemorrhage after CS, myomectomy and hemorrhage from anterior abdominal wound dehiscence. The main surgeries performed were: hysterectomy, ligation of ascending branches of uterine arteries, ligation of hypogastric arteries, debridement and re-suturing of the uterine incision and secondary suturing of anterior abdominal wall. There were 6 near missed fatalities. There were 4 mortalities caused by excessive hemorrhage and severe sepsis. Authors concluded the case fatality rate for re-laparotomy post-CS was high (9\%) and near missed-fatalities were common [11].

Discussing on scientific literature, there are other few case reports on post-CS hemorrhage [12-15], but not a observational studies on CS complicated by hemorrhage from the incision site, as in the present case, especially on patients undergoing scheduled repeated CSs or for antepartum hemorrhage are more prone to hemorrhage at time of surgery, associated with significant maternal mortality and morbidity.

In our experience, a part the extra hemostatic sutures taken at incision site to control local ooze, we used TachoSil drug, a sterile, ready to use absorbable patch for intraoperative topical application, works by mimicking the final steps of the natural blood clotting process, creating a robust fibrin clot at the surgical wound site to achieve hemostasis and sealing in 3-5 minutes. TachoSil provides quick, reliable hemostasis by creating a robust fibrin clot, which adheres to the tissue surface and it is involved in the sealing of lymphatic capillaries. During this process, thrombin converts fibrinogen into fibrin monomers, which spontaneously polymerize, and endogenous factor XIII catalyses the cross-linking of fibrin, creating a firm mechanically stable network. The resulting fibrin clot ensures effective hemostasis and sealing, thus preventing leaks. The cross-linking of fibrin creates a robust matrix with the collagen carrier, providing sealing as well as giving mechanical support to the tissue [16]. It has a large patch of $9.5 \mathrm{~cm} \mathrm{x} 4.8 \mathrm{~cm} \mathrm{x} 0.5$ $\mathrm{cm}$, a medium patch of $4.8 \mathrm{~cm} \mathrm{x} 4.8 \mathrm{~cm} \times 0.5 \mathrm{~cm}$ and a small patch of 3.0 $\mathrm{cm} \times 2.5 \mathrm{~cm}$ x $0.5 \mathrm{~cm}$. At our Hospital, TachoSil is currently available for clinical practice and it is distributed by the hospital pharmacy and this product is currently approved in Europe for supportive haemostatic treatment in surgery, since the efficacy and safety of TachoSil was already demonstrated in many surgeries [17-21].

A study affirmed that postoperative fluid collections are common after CS and hysterectomy, but there are not associated with postoperative morbidity [22].

The scientific literature reports the early benefit of the not suturing visceral peritoneum during CS, but in rare cases possible haemorrhagic complications complicate the operation, since the uterine incision inadequate haemostasis creates a bleeding that decants from the prevesical vessels in the large peritoneal cavity, causing a hemoperitoneum, in $24-36 \mathrm{~h}[6,7,23,24]$.

In the presence of clinical signs of hemoperitoneum a US evaluation of the abdominal cavity is urgent to confirm the presence of the free fluid and the treatment depends by the hemoperitoneum level: it could be done by trans-vaginal or trans-abdominal needle aspiration in slight cases or, in other cases, it is possible to utilize laparoscopy to drain the blood and to suture the bleeding zone of the uterine incision. When the local hemorrhage produce a hemoperitoneum it is mandatory an immediate re-laparotomy to check the LUS hemostasis by further stitches and hemostatic agents, as in our case. We covered the uterine incision site with the Tachosil drug and stitched it there with the successful control of blood loss from the incision site. Thus, by this equine collagen patch coated with human coagulation factors appears to be a safe and effective modality for post-LUS incision hemorrhage. However, further prospective studies are advised before recommending its routine use for uterine incision hemorrhage.

\section{Conclusion}

An inadequate hemostasis of the uterine incision and the vesicaluterine vessels during CS, in closed and open visceral peritoneum, could lead to early hemorrhagic compliances, to control by further surgery. Based on this author's experiences, the collagen patch coated with the human coagulation factors, the TachoSil device, in preventing post-CS LUS hemorrhage seems to have been shown. Even if TachoSil seems to provide a useful additional treatment option in reducing post-CS LUS bleeding, my investigation is jus a case report, but further more extensive study using TachoSil on a larger number of patients operated by repeated CSs, with their Quality of Life and cost analysis evaluation, as useful adjunct treatment in preventing symptomatic and asymptomatic post-operative blood collection, is needed.

\section{Acknowledgements}

I thank Dr. Cristiano Alex De Marzi, Dr. Amleto Buia and Dr. Fabrizio Totaro Aprile for supplying patient's clinical documents and as surgeons of first and repeated cesarean sections. Author is thankful to Nycomed (Nycomed International Management $\mathrm{GmbH}$, Zurich, Switzerland) for supplying part of scientific literature. However, no other financial assistance was provided by the company. There is no conflict of interest and the results have not been influenced by them.

\section{References}

1. Zizza A, Tinelli A, Malvasi A, Barbone E, Stark M, et al. (2011) Cesarean section in the world: a new ecological approach. J Prev Med Hyg 52: [in press]

2. Mozurkewich EL, Hutton EK (2000) Elective repeat cesarean delivery versus trial of labor: a meta-analysis of the literature from1989 to 1999. Am J Obstet Gynecol 183: 1187-1197.

3. Seal SL, Kamilya G, Bhattacharyya SK, Mukherji J, Bhattacharyya AR (2007) Relaparotomy after cesarean delivery: experience from an Indian teaching hospital. J Obstet Gynaecol Res 33: 804-809.

4. Malvasi A, Tinelli A, Guido M, Cavallotti C, Dell'edera D, et al. (2011) Effect of avoiding bladder flap formation in caesarean section on repeat caesarean delivery. Eur J Obstet Gynecol Reprod Biol 159: 300-304.

5. Blanchette $\mathrm{H}$ (2011) The rising cesarean delivery rate in America: what are the consequences? Obstet Gynecol 118: 687-690.

6. Malvasi A, Tinelli A, Tinelli R, Cavallotti C, Farine D (2008) The diagnosis and management of post-cesarean section hemorrhagic shock. J Matern Fetal Neonatal Med 21: 487-491.

7. Malvasi A, Tinelli A, Tinelli R, Serio G, Pellegrino M, et al. (2008) Subfascia hematomas and hemoperitoneum after cesarean section: prevalence according to closure and non-closure of the parietal peritoneum. Gynecol Obstet Invest 66: 162-168.

8. Shearer EL (1993) Cesaran section: medical benefits and costs. Soc Sci Med 37: 1223-1231.

\section{ACOG (2000) Evaluation of Cesarean Delivery. Washington, DC: ACOG.}

10. Seal SL, Kamilya G, Bhattacharyya SK, Mukherji J, Bhattacharyya AR (2007) Relaparotomy after cesarean delivery: experience from an Indian teaching hospital. J Obstet Gynaecol Res 33: 804-809.

11. Seffah JD (2005) Re-laparotomy after Cesarean section. Int J Gynaecol Obste 88: $253-257$ 
Citation: Tinelli A (2011) Post-Cesarean Section Hemorrage Treated by a Collagen Patch Coated with the Human Coagulation Factors. J Clinic Case Reports 1:e103. doi:10.4172/2165-7920.1000e103

12. Pollio F, Staibano S, De Falco M, Buonocore U, De Rosa G, et al. (2007) Severe secondary postpartum hemorrhage 3 weeks after cesarean section: alternative etiologies of uterine scar non-union. J Obstet Gynaecol Res 33: 360-362.

13. Hidar S, Jerbi M, Hafsa A, Slama A, Bibi M, et al. (2007) The effect of uterine incision expansion at caesarean delivery on perioperative haemorrhage: a prospective randomised clinical trial. Rev Med Liege 62: 235-238.

14. Wagner MS, Bédard MJ (2006) Postpartum uterine wound dehiscence: a case report. J Obstet Gynaecol Can 28: 713-715.

15. Sharma JB, Malhotra M (2006) Successful management of uterine incision hemorrhage in caesarean section with topical oxidized regenerated cellulose (Surgicel Nu Knit): a case report. Arch Gynecol Obstet 274: 115-116.

16. Rickenbacher A, Breitenstein S, Lesurtel M, Frilling A (2009) Efficacy of TachoSil a fibrin-based haemostat in different fields of surgery- a systematic review. Expert Opin Biol Ther 9: 897-907.

17. Czerny M, Fleck T, Salat A, Zimpfer D, Klepetko W, et al. (2004) Sealing of the mediatinum with a local hemostyptic agent reduces chest tube drain duration after complete mediastinal lymph node dissection for stage I and II non-small cell lung carcinoma. Ann Thorac Surg 77: 1028-1032.

18. Frilling A, Stavrou GA, Mischinger HJ, de Hemptinne B, Rokkjaer M, et al. (2005) Effectiveness of a new carrier-bound fibrin sealant versus argon beamer as haemostatic agent during liver resection: a randomised prospective trial.
Langenbecks Arch Surg 390: 114-120.

19. Anegg U, Lindenmann J, Matzi V, Smolle J, Maier A, et al. (2007) Efficiency of fleecebound sealing (TachoSil) of air leaks in lung surgery: a prospective randomised trial. Eur J Cardiothorac Surg 25: 198-202.

20. Siemer S, Lahme S, Altziebler S, Machtens S, Strohmaier W, et al. (2007) Efficacy and safety of Tachosil as hemostatic treatment versus standard suturing in kidney tumor resection: a randomised prospective study. Eur Urol 52: $1156-1163$.

21. Tinelli A, Giorda G, Manca C, Pellegrino M, Prudenzano R, et al. (2011) Prevention of lymphocele in female pelvic lymphadenectomy by a collagen patch coated with the human coagulation factors: A pilot study. J Surg Oncol doi: 10.1002/jso.22110. [Epub ahead of print]

22. Tinelli A, Malvasi A, Vittori G (2009) Laparoscopic treatment of post-cesarean section bladder flap hematoma: A feasible and safe approach. Minim Invasive Ther Allied Technol 18: 356-360.

23. Malvasi A, Tinelli A, Guido M, Zizza A, Farine D, et al. (2010) Should the visceral peritoneum at the bladder flap closed at cesarean sections? A postpartum sonographic and clinical assessment. J Matern Fetal Neonatal Med 23: $662-669$.

24. Malvasi A, Tinelli A, Pacella E (2010) Mass closure of visceral peritoneum at cesarean section. A proposal method. J Matern Fetal Neonatal Med 23: 345346 . 